\title{
Kinetics of procalcitonin and C-reactive protein and the relationship to postoperative infection in young infants undergoing cardiovascular surgery
}

\author{
Jesse Davidson', Suhong Tong ${ }^{2}$, Amanda Hauck', D. Scott Lawson', Eduardo da Cruz ${ }^{1}$ and Jon Kaufman'
}

BACKGROUND: The utility of procalcitonin (PCT) and C-reactive protein (CRP) as infectious biomarkers following infant cardiothoracic surgery is not well defined.

METHODS: We designed a prospective cohort study to evaluate PCT and CRP after infant cardiothoracic surgery. PCT and CRP were drawn preoperatively and $24 / 72 \mathrm{~h}$ postoperation or daily in delayed sternal closure patients. Presence of infection within $10 \mathrm{~d}$ of surgery, vasoactive-inotropic scores at 24 and $72 \mathrm{~h}$, and length of intubation, intensive care unit stay, and hospital stay were documented.

RESULTS: PCT and CRP were elevated at $24 \mathrm{~h}$. PCT then decreased while CRP increased in patients undergoing delayed sternal closure or cardiopulmonary bypass. In the delayed sternal closure group, PCT was significantly higher on postoperative days 2-5 in patients who ultimately developed infection. Higher PCT was independently associated with increased vasoactive-inotropic score at $72 \mathrm{~h}$. CRP did not correlate with infection or postoperative support.

CONCLUSION: PCT rises after cardiothoracic surgery in infants but decreases by $72 \mathrm{~h}$ while CRP remains elevated. Sternal closure may affect CRP but not PCT. PCT is independently associated with circulatory support requirements at $72 \mathrm{~h}$ postoperation and with development of infection. РСТ may have greater utility as a biomarker in this population.

A s defined by the National Institutes of Health in 2001, a biomarker is "a characteristic that can be measured objectively and evaluated as an indicator of normal biological processes, pathologic processes, or pharmacologic responses to a therapeutic intervention" (1). Biomarkers can be used to aid in diagnosis, therapeutic monitoring, and risk stratification. One active area of clinical biomarker research involves the differentiation of sepsis from other causes of the systemic inflammatory response syndrome (SIRS).

Cardiac surgery and cardiopulmonary bypass are potent inducers of SIRS (2). Cardiac surgery patients are also at high risk for infection. Differentiation of noninfectious SIRS from sepsis in this population can be challenging, making the use of sepsis biomarkers intriguing. Currently available biomarkers (white blood cell count with differential and C-reactive protein (CRP)) are inadequate for the evaluation of sepsis in this population (3-8).

Recent research suggests that procalcitonin (PCT) may be useful for this purpose after cardiopulmonary bypass. PCT is the pro-hormone of calcitonin and is produced by neuroendocrine cells of the thyroid and lung. PCT is normally present at extremely low levels in the systemic circulation but increases dramatically following certain exposures $(9,10)$. PCT shows very favorable biomarker kinetics, with a rapid rise following an appropriate exposure, early peak levels, and a rapid decline following treatment or removal of the underlying trigger. PCT may be better than white blood cell count and CRP at differentiating sepsis and SIRS in certain clinical settings, including following cardiac surgery in older children $(3,5)$.

It is unclear whether sepsis biomarkers are as useful in younger cardiac surgery patients. Several prior studies of PCT and CRP after pediatric cardiothoracic surgery have included neonates and infants, but always in combination with older children (5-8,11-15). Younger patients have immature immune responses and tend to have more complex surgeries, including longer bypass/aortic cross clamp times and the use of deep hypothermic circulatory arrest. They frequently require prolonged cardiovascular and respiratory support, and in some cases may be left with an open chest requiring a second operation to close the sternum (delayed sternal closure (DSC)). This combination of factors places them at high risk for both SIRS and sepsis and makes them an ideal patient population for use of an effective sepsis biomarker.

The primary goal of our study was to assess the kinetics of PCT and CRP in neonates and young infants undergoing cardiothoracic surgery. Secondary objectives included observing the effects of DSC on PCT and CRP levels and determining the relationship of PCT and CRP levels to postoperative support and the development of infection. We hypothesized that PCT would demonstrate more favorable kinetics than CRP, and that PCT would have a stronger association with infection and postoperative support requirements. 


\section{Articles | Davidsonet al.}

\section{RESULTS}

A flowchart of enrollment is presented in Figure 1. A total of 70 patients were enrolled in the study between July 2009 and September 2010. One patient was found to have an inaccurate date of birth in the medical record, was older than inclusion criteria permitted, and was excluded from all subsequent analyses. Of the remaining 69 patients, 55 underwent cardiopulmonary bypass, of which 26 required DSC. One additional patient did not undergo cardiopulmonary bypass but required DSC owing to shunt malfunction and immediate reoperation for shunt revision. Patients receiving neither cardiopulmonary bypass nor DSC are termed "non-bypass" for the remainder of this report. All patients completed the study protocol.

Demographic and baseline data for the cohort have been previously published (Table 1) (16). Overall, the DSC and nonbypass patients were younger than the general cardiopulmonary bypass patients, and the DSC patients weighed significantly less at the time of operation. The DSC group had higher Aristotle scores, a higher prevalence of single ventricle physiology, and greater use of preoperative steroids. Median bypass time and deep hypothermic circulatory arrest time were also longer in the DSC, although aortic cross clamp time was comparable.

Median preoperation, $24 \mathrm{~h}$, and $72 \mathrm{~h}$ PCT and CRP levels are shown in Table 2. Preoperative PCT and CRP levels were normal in all groups with the exception of the non-bypass group, which demonstrated borderline elevations in both PCT and CRP. Following surgery, PCT and CRP levels were elevated above normal in all groups. Mean change in PCT and CRP levels from preoperation to 24 and $72 \mathrm{~h}$ are shown in Table 3 . PCT levels at $24 \mathrm{~h}$ were significantly higher than baseline in the DSC and cardiopulmonary bypass groups with a nonsignificant increase seen in the non-bypass group. CRP levels were

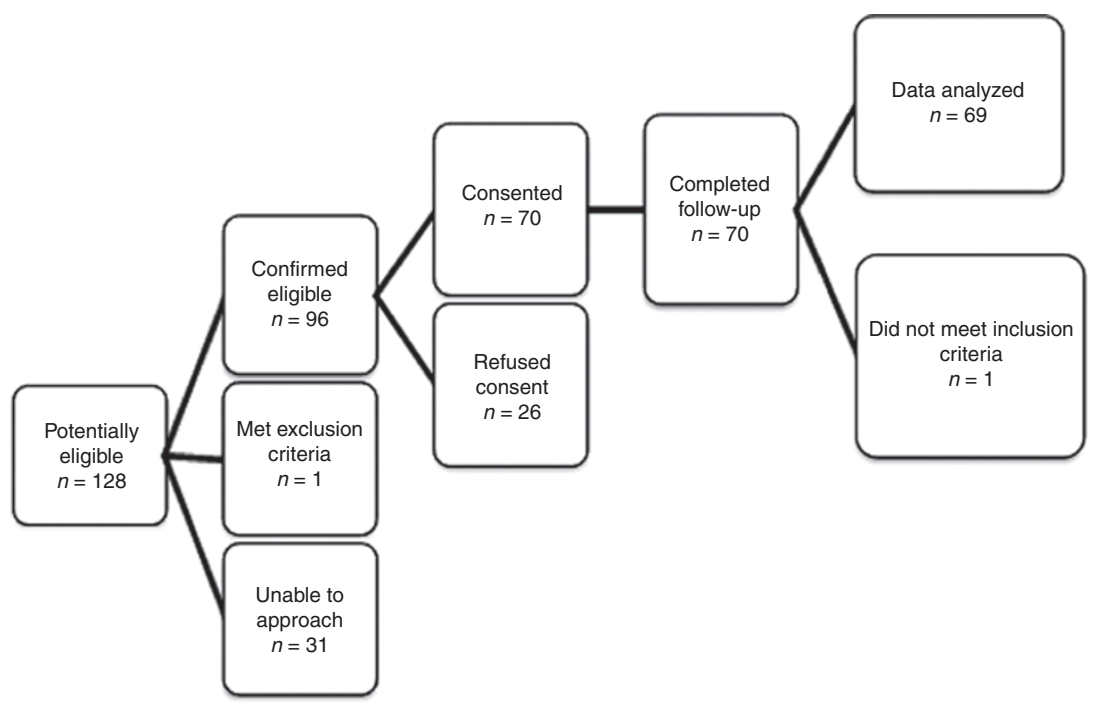

Figure 1. Recruitment flowchart.

Table 1. Baseline demographics and surgical data

\begin{tabular}{|c|c|c|c|c|c|c|}
\hline & $\begin{array}{c}\text { No CPB, } \\
n=13\end{array}$ & $\begin{array}{c}\mathrm{CPB} \\
n=29\end{array}$ & $\begin{array}{l}\text { DSC, } \\
n=27\end{array}$ & $\begin{array}{c}P \text { value } \\
\text { (no CPB vs. CPB) }\end{array}$ & $\begin{array}{c}P \text { value } \\
\text { (no CPB vs. DSC) }\end{array}$ & $\begin{array}{c}\text { Pvalue } \\
\text { (CPB vs. DSC) }\end{array}$ \\
\hline Male, $n(\%)$ & $8(62 \%)$ & $16(55 \%)$ & $18(67 \%)$ & ns & ns & ns \\
\hline Ethnicity, $n(\%)$ & & & & ns & ns & ns \\
\hline White & $9(69 \%)$ & $18(62 \%)$ & $15(56 \%)$ & & & \\
\hline Hispanic & $4(31 \%)$ & $10(34 \%)$ & $11(40 \%)$ & & & \\
\hline Other & $0(0 \%)$ & $1(4 \%)$ & $1(4 \%)$ & & & \\
\hline Age in days, median (range) & $8(2-54)$ & $49(3-90)$ & $5(2-81)$ & $<0.001$ & ns & $<0.005$ \\
\hline Weight (kg) & $3.3(2.4-4.2)$ & $3.4(2.6-5.2)$ & $3.1(2.3-3.7)$ & ns & ns & $<0.005$ \\
\hline Aristotle score median (range) & $8(6-13)$ & $10(6-18 \cdot 1)$ & $11(6.3-17.5)$ & $<0.05$ & $<0.001$ & $<0.005$ \\
\hline Bypass time (min), median (range) & $\mathrm{n} / \mathrm{a}$ & $130(61-213)$ & $168.5(88-316)$ & $\mathrm{N} / \mathrm{A}$ & $\mathrm{N} / \mathrm{A}$ & $<0.05$ \\
\hline Cross clamp time (min), median (range) & $\mathrm{n} / \mathrm{a}$ & $86(28-248)$ & $77(31-440)$ & $\mathrm{N} / \mathrm{A}$ & $\mathrm{N} / \mathrm{A}$ & ns \\
\hline DHCA time (min), median (range) & $\mathrm{n} / \mathrm{a}$ & $0(0-110)$ & $20(0-72)$ & $\mathrm{N} / \mathrm{A}$ & $\mathrm{N} / \mathrm{A}$ & $<0.01$ \\
\hline Single ventricle physiology & $3(23 \%)$ & $2(7 \%)$ & $12(44 \%)$ & ns & ns & $<0.005$ \\
\hline Preoperative steroids & $1(8 \%)$ & $12(41 \%)$ & $23(85 \%)$ & $<0.05$ & $<0.001$ & $<0.005$ \\
\hline
\end{tabular}

CPB, cardiopulmonary bypass; DHCA, deep hypothermic circulatory arrest; DSC, delayed sternal closure; N/A, not applicable; ns, not significant. 
Table 2. Preoperative, $24 \mathrm{~h}$, and $72 \mathrm{~h} \mathrm{PCT}$ and CRP levels

\begin{tabular}{|c|c|c|c|c|c|c|}
\hline & No CPB & CPB & DSC & $\begin{array}{c}P \text { value } \\
\text { (no CPB vs. CPB) }\end{array}$ & $\begin{array}{c}P \text { value } \\
\text { (no CPB vs. DSC) }\end{array}$ & $\begin{array}{c}P \text { value } \\
\text { (CPB vs. DSC) }\end{array}$ \\
\hline $\begin{array}{l}\text { PCT preoperation }(\mathrm{ng} / \mathrm{ml}) \text { median } \\
\text { (range) }\end{array}$ & $0.5(0.13-6.35)$ & $0.11(0.10-1.35)$ & $0.20(0.10-3.96)$ & $<0.001$ & $<0.01$ & 0.085 \\
\hline РCT 24h (ng/ml) median (range) & $1.10(0.10-3.98)$ & $2.45(0.12-137.10)$ & $3.10(0.32-55.94)$ & $<0.05$ & $<0.05$ & ns \\
\hline PCT $72 \mathrm{~h}(\mathrm{ng} / \mathrm{ml})$ median (range) & $0.28(0.16-4.56)$ & $1.21(0.10-35.96)$ & $1.73(0.11-57.40)$ & $<0.01$ & $<0.005$ & ns \\
\hline CRP preoperation (mg/dl) mean (SD) & $0.96(0.74)$ & $0.38(0.24)$ & $0.37(0.32)$ & $<0.001$ & $<0.001$ & ns \\
\hline CRP $72 \mathrm{~h}(\mathrm{mg} / \mathrm{dl})$ mean $(\mathrm{SD})$ & $3.21(1.89)$ & $7.57(4.87)$ & $5.95(4.81)$ & $<0.05$ & ns & ns \\
\hline
\end{tabular}

CPB, cardiopulmonary bypass; CRP, C-reactive protein; DSC, delayed sternal closure; ns, not significant; PCT, procalcitonin.

Table 3. Change in biomarker levels from preoperation to $72 \mathrm{~h}$ after surgery

\begin{tabular}{lcccc}
\hline & $\begin{array}{c}24 \mathrm{~h} \text { vs. preoperation } \\
(\mathrm{ng} / \mathrm{ml}) \text {, mean (SD) }\end{array}$ & $P$ value & $\begin{array}{c}72 \mathrm{vs} .24 \mathrm{~h} \\
(\mathrm{ng} / \mathrm{ml}) \text {, mean (SD) }\end{array}$ & $P$ value \\
\hline PCT & & & & \\
No CPB & $0.27(1.42)$ & $\mathrm{ns}$ & $-0.97(1.30)$ & $<0.05$ \\
CPB & $13.50(32.17)$ & $<0.001$ & $-9.59(23.59)$ & $<0.001$ \\
DSC & $8.99(16.06)$ & $<0.001$ & $-3.39(7.98)$ & $<0.01$ \\
CRP & & & & \\
No CPB & $4.24(3.04)$ & $<0.001$ & $-2.12(1.65)$ & $<0.005$ \\
CPB & $5.53(3.06)$ & $<0.001$ & $1.68(4.17)$ & $<0.05$ \\
DSC & $3.49(2.08)$ & $<0.001$ & $2.66(4.36)$ & $<0.01$ \\
\hline
\end{tabular}

CPB, cardiopulmonary bypass; CRP, C-reactive protein; DSC, delayed sternal closure; $\mathrm{PCT}$, procalcitonin.

elevated above baseline in all groups. By $72 \mathrm{~h}$ after the operation, PCT levels had fallen significantly in all groups. CRP levels also fell in the non-bypass group, but rose significantly in the DSC and cardiopulmonary bypass groups.

Daily PCT and CRP levels were obtained in the DSC group in order to assess the effects of DSC on these inflammatory biomarkers. Median levels and interquartile ranges are shown in Figure 2. Comparing biomarker levels from the day of chest closure to the day following chest closure, PCT levels showed a small, statistically significant mean decrease $(-0.28 \mathrm{ng} / \mathrm{ml}$; SD: 0.67; $P<0.0001$ ), whereas CRP levels trended higher (mean increase $1.36 \mathrm{mg} / \mathrm{dl}$; SD: 3.91; $P=0.11$ ).

Postoperative support requirements for each group are shown in Table 4. As previously published (16), the DSC group required significantly more postoperative support as demonstrated by higher median vasoactive-inotropic score (VIS) (17) at 24, 48, and $72 \mathrm{~h}$ postoperation, and longer median intubation time, cardiac intensive care unit (CICU) stay, and hospital stay. Spearman correlation testing was utilized to assess for associations between PCT and CRP at 24 and $72 \mathrm{~h}$ and postoperative support. PCT at $72 \mathrm{~h}$ showed a statistically significant moderate positive correlation with VIS at 24 and $72 \mathrm{~h}(r=0.48, P<0.0001 ; r=0.44, P<0.0005)$, as well as weaker correlations with intubation time $(r=0.40, P<0.005)$, peak lactate $(r=0.40 ; P<0.005)$, and length of CICU $(r=0.39$; $P<0.005)$, and hospital stay $(r=0.29 ; P<0.05)$. PCT at $24 \mathrm{~h}$


Figure 2. Daily levels of (a) procalcitonin (PCT) and (b) C-reactive protein (CRP) in patients undergoing delayed sternal closure (DSC). Box $=25-75 \%$; line inside box = median; cross = mean; small box = outlier.

was also weakly correlated with VIS at $24 \mathrm{~h}(r=0.40, P<0.005)$ and $72 \mathrm{~h}(r=0.31, P<0.05)$, intubation time $(r=0.29, P<$ $0.05)$, and peak lactate $(r=0.30 ; P<0.05)$. CRP did not demonstrate a statistically significant positive correlation with any measure of postoperative support.

Next, we used linear regression modeling to analyze the association of PCT and CRP on short-term surgical outcomes 


\section{Articles $\mid$ Davidson et al.}

Table 4. Short-term surgical outcomes

\begin{tabular}{|c|c|c|c|c|c|c|}
\hline Outcome variable & $\begin{array}{c}\text { No CPB } \\
n=13\end{array}$ & $\begin{array}{c}\text { CPB, } \\
n=29\end{array}$ & $\begin{array}{c}\text { DSC, } \\
n=27\end{array}$ & $\begin{array}{c}P \text { value } \\
\text { (no CPB vs. } \\
\text { CPB) }\end{array}$ & $\begin{array}{c}P \text { value } \\
\text { (no CPB vs. } \\
\text { DSC) }\end{array}$ & $\begin{array}{c}\text { Pvalue } \\
\text { (CPB vs. DSC) }\end{array}$ \\
\hline Intubation, hours, median (range) & $27.5(8,99)$ & $37(4,148)$ & $117(50,410)$ & ns & $<0.001$ & $<0.001$ \\
\hline Length of hospital stay, days, median (range) & $11.5(2,81)$ & $8.5(4,36)$ & $21(7,106)$ & ns & $<0.01$ & $<0.001$ \\
\hline Length of CICU stay, days, median (range) & $3(1,23)$ & $4(1,11)$ & $8.5(4,31)$ & ns & $<0.05$ & $<0.01$ \\
\hline VIS24, median (range) & $0(0,8)$ & $8(0,20)$ & $15(8,23.5)$ & $<0.05$ & $<0.001$ & $<0.001$ \\
\hline VIS72, median (range) & $0(0,10)$ & $0(0,15)$ & $10.5(3,31)$ & ns & $<0.001$ & $<0.001$ \\
\hline Peak lactate $(\mathrm{mmol} / \mathrm{l})$ & $2.3(0.9,4.8)$ & $3.7(1.0,15.3)$ & $7.1(2.9,15.1)$ & $<0.05$ & $<0.001$ & $<0.001$ \\
\hline
\end{tabular}

CICU, cardiac intensive care unit; CPB, cardiopulmonary bypass; DSC, delayed sternal closure; ns, not significant; VIS, vasoactive-inotropic score.

Table 5. Generalized linear regression modeling of biomarker effects on short-term surgical outcomes

\begin{tabular}{|c|c|c|c|c|c|c|}
\hline & $\begin{array}{c}\text { VIS at } 24 \mathrm{~h}, \\
\text { nean }(\mathrm{SE}) ; P \text { value }\end{array}$ & $\begin{array}{c}\text { VIS at } 72 \mathrm{~h} \\
\text { mean }(\mathrm{SE}) ; P \text { value }\end{array}$ & $\begin{array}{l}\text { Length of intubation } \\
\text { (hours), mean (SE); } \\
\text { Pvalue }\end{array}$ & $\begin{array}{l}\text { Peak lactate } \\
(\mathrm{mmol} / \mathrm{l}), \text { mean } \\
(\mathrm{SE}) ; P \text { value }\end{array}$ & $\begin{array}{l}\text { Length of ICU stay } \\
\text { (days), mean (SE); } \\
\qquad \text { value }\end{array}$ & $\begin{array}{l}\text { Length of hospital } \\
\text { stay (days), mean } \\
\text { (SE); } P \text { value }\end{array}$ \\
\hline РCT $24 \mathrm{~h}$ & $0.03(0.03) ; \mathrm{ns}$ & $0.04(0.003) ; \mathrm{ns}$ & 1.01 (1.00); ns & $0(0.02) ; \mathrm{ns}$ & $1.00(1.00) ; \mathrm{ns}$ & $1.00(1.00) ; \mathrm{ns}$ \\
\hline РСТ $72 \mathrm{~h}$ & $0.10(0.16) ; 0.11$ & $0.16(0.07) ;<0.05$ & $1.02(1.01) ; 0.076$ & $-0.02(0.04) ; \mathrm{ns}$ & 1.01 (1.01); ns & $1.00(1.01) ; \mathrm{ns}$ \\
\hline CRP $24 \mathrm{~h}$ & $-0.09(0.23) ; \mathrm{ns}$ & $0.01(0.26) ; \mathrm{ns}$ & 0.98 (1.04); ns & $-0.09(0.14) ; \mathrm{ns}$ & $1.00(1.04) ; \mathrm{ns}$ & $1.02(1.03) ; \mathrm{ns}$ \\
\hline CRP $72 \mathrm{~h}$ & $-0.03(0.14) ; \mathrm{ns}$ & $-0.02(0.15) ; \mathrm{ns}$ & 1.00 (1.02); ns & $0.02(0.08) ; \mathrm{ns}$ & 1.00 (1.02); ns & 1.00 (1.02); ns \\
\hline
\end{tabular}

CRP, C-reactive protein; ICU, intensive care unit; ns, not significant; PCT, procalcitonin; VIS, vasoactive-inotropic score.

while controlling for surgical group and preoperative PCT and CRP levels (Table 5). Surgical group had a strong effect on all outcomes measured. Despite this, modeling demonstrated an independent association of PCT at $72 \mathrm{~h}$ on VIS at $72 \mathrm{~h}(0.16$ increase in VIS per $1 \mathrm{ng} / \mathrm{ml}$ increase in PCT $\left(P=0.02, R^{2}=\right.$ $0.57)$ ), with a trend toward independent associations with VIS at $24 \mathrm{~h}\left(P=0.11, R^{2}=0.59\right)$ and intubation time in hours $\left(P=0.08, R^{2}=0.57\right)$. PCT at $24 \mathrm{~h}$ and CRP at any time point assessed were not independently associated with the surgical outcomes measured.

Infection rates and types by surgical group are shown in Table 6. Given the low rate of infection in the non-bypass and cardiopulmonary bypass groups, statistical analysis of the relation of inflammatory biomarkers to infection was limited to the DSC group. The trends of PCT and CRP levels in DSC patients who developed infections in the first ten postoperative days vs. those who did not are shown in Figure 3. Baseline PCT and CRP levels were similar in both groups. By $48 \mathrm{~h}$ after the operation, PCT was significantly lower in the patients who did not develop infection compared with those who did, and this difference persisted through postoperative day 5 (Table 7). The difference was in large part owing to a rapid decrease in PCT levels in the noninfected group compared with a relative plateau in patients who developed infections (Figure 3). $\mathrm{CRP}$ levels increased through $72 \mathrm{~h}$ followed by a slow decrease (Figure 3) and did not differentiate between infected and noninfected patients in the first five postoperative days (Table 7).

\section{DISCUSSION}

The primary purpose of our study was to assess the kinetics of PCT and CRP in infants $\leq 90 \mathrm{~d}$ of age undergoing cardiothoracic
Table 6. Infection type and frequency

\begin{tabular}{lccc}
\hline Infection type & No CPB $(n=13)$ & CPB $(n=29)$ & DSC $(n=27)$ \\
\hline Bloodstream & $0(0 \%)$ & $0(0 \%)$ & $2(7 \%)$ \\
Urinary tract & $0(0 \%)$ & $0(0 \%)$ & $1(4 \%)$ \\
Wound & $1(8 \%)$ & $1(3 \%)$ & $5(19 \%)$ \\
Trachea & $0(0 \%)$ & $1(3 \%)$ & $1(4 \%)$ \\
Pneumonia & $0(0 \%)$ & $0(0 \%)$ & $0(0 \%)$ \\
Other & $1(8 \%)$ & $0(0 \%)$ & $1(4 \%)$ \\
Total & $2(15 \%)$ & $2(7 \%)$ & $9(33 \%)^{\mathrm{a}}$
\end{tabular}

$\mathrm{CPB}$, cardiopulmonary bypass; DSC, delayed sternal closure.

ane patient presented with simultaneous urinary tract infection and bacteremia.

surgery. We found that both PCT and CRP levels predictably increased by postoperative day 1 in our population, making them poor tools for the diagnosis of infection within the first $24 \mathrm{~h}$ after cardiothoracic surgery. However, in agreement with prior studies in adults and older children $(3,6-8)$, we found that PCT peaked at $24 \mathrm{~h}$ then fell sharply by $72 \mathrm{~h}$, whereas CRP continued to rise through $72 \mathrm{~h}$ except in non-bypass patients. These findings suggest that kinetics favor the use of PCT over CRP for the identification of infections after postoperative day 1.

Adult studies have also suggested the possibility of using cutoffs to differentiate elevations in PCT due to SIRS vs. sepsis (3). Postoperative increases in PCT in adults were generally small $(0.5-7 \mathrm{ng} / \mathrm{ml})(3)$ and close to suggested cut-offs for identifying bacterial infections in other settings $(0.5-2 \mathrm{ng} / \mathrm{ml})(18-20)$. By contrast, Michalik et al. and Hammer et al. reported occasional large increases in PCT $(>50 \mathrm{ng} / \mathrm{ml})$ in pediatric 


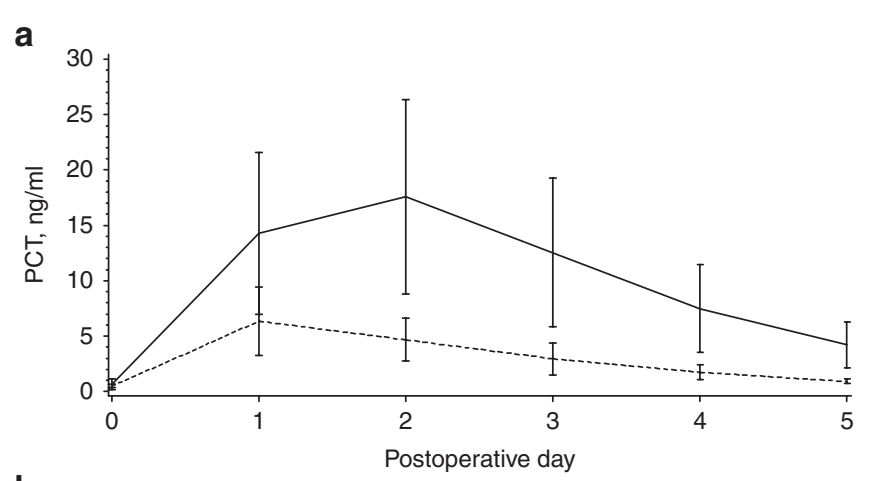

b

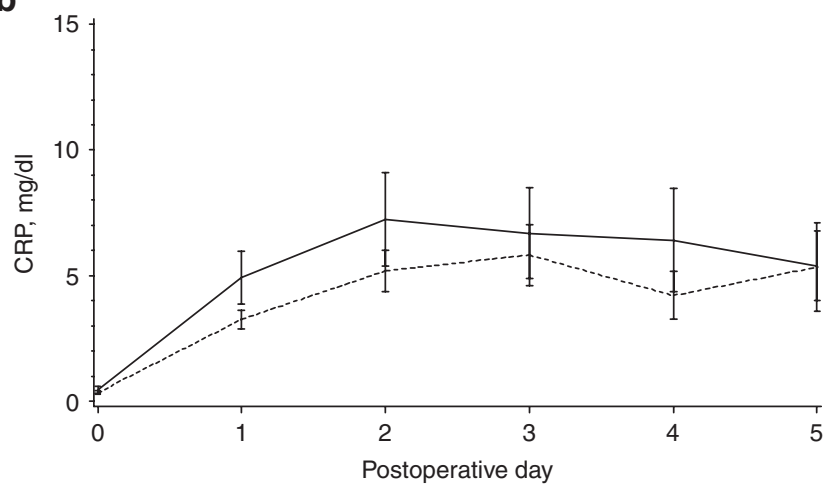

Figure 3. Trends in (a) mean procalcitonin (PCT) and (b) mean C-reactive protein (CRP) levels in infected vs. noninfected patients. Solid line $=$ infection; dashes $=$ no infection; bars $=$ SE.

Table 7. Difference in biomarker levels in delayed sternal closure patients who developed infection in the first 10 postoperative days vs. noninfected DSC patients

\begin{tabular}{lccccc}
\hline & \multicolumn{5}{c}{ Biomarker mean difference $(P$ value) } \\
\cline { 2 - 6 } & POD 1 & POD 2 & POD 3 & POD 4 & POD 5 \\
\hline PCT & 2.33 & 3.41 & 3.97 & 3.28 & 4.61 \\
$(\mathrm{ng} / \mathrm{ml})$ & $(0.139)$ & $(<0.05)$ & $(<0.05)$ & $(<0.05)$ & $(<0.005)$ \\
CRP & 1.63 & 3.13 & 1.68 & 1.84 & 2.59 \\
$(\mathrm{mg} / \mathrm{dl})$ & $(0.055)$ & $(0.105)$ & $(\mathrm{ns})$ & $(\mathrm{ns})$ & $(\mathrm{ns})$ \\
\hline
\end{tabular}

CRP, C-reactive protein; DSC, delayed sternal closure; ns, not significant; PCT, procalcitonin; $\mathrm{POD}$, postoperative day.

patients undergoing cardiothoracic surgery with cardiopulmonary bypass $(12,15)$. Our study identified a substantial subset of patients (13\%) who developed very high PCT levels (>20 ng/ $\mathrm{ml}$ ) by $24 \mathrm{~h}$ postoperation. These high peak levels suggest that it is impractical to utilize absolute PCT values to differentiate between SIRS and sepsis in this patient population. However, the consistent and rapid decrease in PCT levels by $72 \mathrm{~h}$ regardless of the peak value still allows for the use of PCT trend as a marker for possible infection in the early postoperative period.

Given the need to utilize biomarker trends to screen for infection, we were concerned that DSC might interfere with interpretation of PCT and CRP levels. Specifically, it was possible that the second surgical procedure could cause an additional increase in inflammatory markers, mimicking the "double peak" of potential infection. We therefore sought to compare PCT and CRP levels on the day of chest closure to levels obtained on the day following chest closure. Overall, of 27 patients, PCT levels decreased in 24 following chest closure, but remained unchanged in two patients and increased in one, leading to a small but statistically significant mean decrease in PCT. CRP levels rose following chest closure in 16 of 24 patients but fell in 7 patients, leading to a trend towards increased CRP. Of note, in 7 patients CRP levels had initially peaked then increased again following chest closure, leading to the appearance of a double peak. Based on these results, PCT should be expected to decline following chest closure, and an increase should be considered abnormal. An increase in CRP following chest closure is common and should not automatically prompt a search for secondary causes, even in the setting of a double peak.

As a secondary goal, we sought to evaluate PCT and CRP levels in the subgroup of our patients who developed infections after surgery. Several previous studies have investigated this relationship in older children undergoing cardiac surgery. Two studies demonstrated markedly better areas under the receiver operating characteristic curve for PCT vs. CRP for the differentiation of sepsis from SIRS in this population $(4,5)$. Optimal PCT cut-offs were $\sim 2 \mathrm{ng} / \mathrm{ml}$ in both cases. A second increase in PCT was also described in children who subsequently developed infection (13). More recently, Nahum et al. performed a large prospective cohort study to evaluate PCT and CRP in febrile postoperative cardiac patients $(14,21)$. They found that both PCT and CRP levels were significantly higher on the day of fever in patients with infection compared with those without, although overlap did occur between the groups. CRP velocity (change in CRP over time) was also higher in patients with infection (PCT velocity data were not reported).

In this study, the majority of infections occurred in the DSC group. By protocol, PCT and CRP levels were followed daily in this group. We trended these biomarkers over the first five postoperative days to assess for differences among patients who developed infections compared with those who did not. By $48 \mathrm{~h}$ postoperation, a statistically significant difference was seen in PCT levels between these groups, and this difference grew over the first five postoperative days. The difference in PCT levels was driven primarily by a rapid decrease in PCT levels in noninfected patients. Over the same time period, there was no significant difference in CRP levels between infected and noninfected patients. Our findings are consistent with prior studies showing that PCT may be more useful than CRP in helping to identify potentially infected patients. In particular, the early divergence of PCT levels may allow for aggressive culture surveillance and/or early treatment before the development of clinically apparent infection. In contrast to the study by Nahum et al., our data indicate that even CRP trend may be difficult to interpret as an early marker of infection in this population (21).

Finally, in our study we have identified a mild independent association between increased PCT levels and higher vasoactive-inotropic support requirements at $72 \mathrm{~h}$, as well as a trend toward prolonged intubation. Although our study was underpowered to completely assess these endpoints, our findings are 


\section{Articles $\quad$ Davidson et al.}

consistent with multiple pediatric and adult studies in sepsis (22), cardiac arrest $(23,24)$, and cardiac surgery $(7,8)$ that demonstrated significantly higher PCT levels in critically ill patients with complications or poor outcomes. CRP does not appear to have a similar correlation in these populations $(3,7,8)$, leading to speculation that PCT itself may have a pathologic role in some cases. Additional support for this hypothesis is given by animal models of sepsis, in which early or late immunoneutralization of PCT led to markedly decreased mortality (10). Further research is needed to clarify whether a direct pathologic role exists for PCT in infants undergoing cardiothoracic surgery.

Our study does have some limitations. First, it represents a single-center experience in a specific, high-risk patient population, potentially limiting the generalizability of our findings. Second, the study design does not differentiate between high PCT levels caused by early subclinical infection vs. levels elevated by other postoperative factors, such as low cardiac output, which might independently increase the risk for infection. Third, the study was powered to evaluate PCT and CRP kinetics rather that the relationship of these biomarkers to infections. As such it was underpowered to establish biomarker cut-offs owing to the relatively low number of infections. Finally, limitations on the volume of blood drawn for laboratory tests did not allow us to draw daily levels in low-risk patients who were unlikely to otherwise require a blood transfusion. It is therefore possible to have missed additional increases in biomarker levels between 24 and $72 \mathrm{~h}$ postoperation in the non-bypass and cardiopulmonary bypass groups.

\section{Conclusions}

PCT and CRP levels are routinely elevated in neonates and young infants $24 \mathrm{~h}$ after cardiothoracic surgery. By $72 \mathrm{~h}$, PCT levels reliably fall but CRP levels do not, making PCT kinetics potentially more favorable than CRP kinetics for infection screening in the early postoperative period. DSC does not further increase PCT but may increase CRP. PCT, but not CRP, may help to identify DSC patients at risk for developing infection as early as postoperative day 2. Higher PCT levels at $72 \mathrm{~h}$ are independently associated with increased circulatory support at $72 \mathrm{~h}$ and a trend toward increased length of intubation. CRP levels are not associated with postoperative support. Based on these findings, PCT may be better than CRP as a sepsis biomarker in this population.

\section{METHODS}

Patients were enrolled in a prospective, observational cohort study examining the postoperative kinetics of PCT and CRP in infants $\leq 90$ $\mathrm{d}$ of age undergoing cardiothoracic surgery. Exclusion criteria were estimated gestational age $<34$ wk or weight $<1,200 \mathrm{~g}$ at the time of surgery. The study was approved by the Colorado Multiple Institutional Review Board. Informed consent was obtained in all cases before enrollment.

Baseline demographic and surgical information collected on all patients included gender, ethnicity, gestational age at delivery, age and weight at the time of surgery, anatomic diagnosis, surgical procedure, Aristotle comprehensive complexity score, use of preoperative steroids, cardiopulmonary bypass time, aortic cross clamp time, and deep hypothermic circulatory arrest time. By protocol, all patients underwent preoperative assessment of PCT, CRP, and liver function tests in the operating room before opening incision. Normal PCT levels were $<0.5 \mathrm{ng} / \mathrm{ml}$ and normal CRP levels were $<1 \mathrm{mg} / \mathrm{dl}$. PCT concentration was measured via a commercially available immunoluminometric assay (PCT sensitive Kryptor System; Brahms Aktiengesllschaft, Annapolis, MD).

Cardiopulmonary bypass was performed using a neonatal circuit consisting of a roller head pump (Terumo System 1; Terumo Cardiovascular Systems, Ann Arbor, MI) and a Terumo FX05 oxygenator with a blood prime. The prime routinely underwent hemofiltration using a Sorin DHFO.6 hemoconcentrator (Sorin Group, Milan, Italy) with a polyethersulfone membrane before initiating bypass, allowing for partial filtration of molecules up to 65,000 Da. Anticoagulation was achieved before bypass by administering 400 units $/ \mathrm{kg}$ of heparin systemically to the patient. Initial target flow rate was approximately $200 \mathrm{ml} / \mathrm{kg} / \mathrm{min}$. Cardioplegia was accomplished using del Nido formula cardioplegia solution at an initial dose of $30 \mathrm{ml} / \mathrm{kg}$ and subsequent dosing considered after 45-60 min of aortic cross clamp time. Conventional ultrafiltration was utilized throughout cardiopulmonary bypass. After bypass, modified ultrafiltration was employed to remove inflammatory mediators and increase hematocrit. Cefazolin (vancomycin if allergic or methicillin-resistant Staphylococcus aureus colonized) was given less than $60 \mathrm{~min}$ before incision (after induction of anesthesia), and redosed after $6 \mathrm{~h}$ or within $1 \mathrm{~h}$ of completion of bypass (whichever was earlier). Two additional doses were given at $8 \mathrm{~h}$ intervals if the sternum was primarily closed or were continued at $8 \mathrm{~h}$ intervals until two doses after chest closure in DSC patients. Neonates (0-28 d) undergoing cardiopulmonary bypass received $30 \mathrm{mg} / \mathrm{kg}$ methylprednisolone at 10 and $4 \mathrm{~h}$ preoperation if inpatients or once during induction if outpatients. Older infants (29-90 d) received this dose on induction if deep hypothermic circulatory arrest was anticipated.

All patients were admitted to the CICU following the operation. At that time, patients were separated into one of three groups based on the following operative characteristics: (i) DSC, (ii) cardiopulmonary bypass without DSC, and (iii) no cardiopulmonary bypass or DSC. The decision to perform DSC was made by the attending surgeon as a precaution in patients with poor function, significant myocardial edema, or other concerns for low cardiac output. Patients returning to the CICU with an open chest had PCT and CRP levels assessed daily until $3 \mathrm{~d}$ following sternal closure. Patients without DSC had PCT and CRP levels assessed at 24 and $72 \mathrm{~h}$ after surgery in order to limit blood volume drawn from patients who were unlikely to require transfusion as part of routine clinical course. Additional laboratory testing was performed as directed by the intensivist.

Invasive arterial and central venous pressure monitoring were performed in all cases. Inotropic and vasoactive medications were initiated at the discretion of the surgical team. Ongoing titration was directed by the intensivist and did not follow a pre-established protocol. VIS was calculated as per Gaies et al. (17) at 24 and $72 \mathrm{~h}$ after surgery, timed with the assessment of PCT and CRP levels.

The primary outcomes determined a priori were the absolute levels of PCT and CRP at 24 and $72 \mathrm{~h}$ after operation. Secondary outcomes were change in PCT and CRP levels before and after DSC, VIS at 24 and $72 \mathrm{~h}$ after surgery, length of intubation in hours, length of CICU and hospital stay, peak lactate concentration, and infection within 10 $\mathrm{d}$ of surgery. Assessment for infection was performed at the discretion of the clinical team. Initiation of antibiotic therapy was documented on all patients. Patients with evidence of SIRS who recovered without antibiotic therapy were deemed to not have clinically significant infections. Patients in whom antibiotic therapies were initiated but discontinued within $48 \mathrm{~h}$ after initial cultures were normal were also considered to have no significant infections. Clinical data were collected on all other patients in whom antibiotics were initiated and used to categorize the patient into one of six different categories: bacteremia, wound infection, tacheitis, pneumonia, urinary tract infection, or other. Bacteremia was diagnosed if SIRS criteria were present in the setting of a positive blood culture. The diagnosis of wound infection required the presence of erythema or dehiscence with purulent drainage with or without deep tissue involvement. Tracheitis was diagnosed if the patient developed fever or worsening respiratory status and either heavy polymorphonuclear leukocytes or pathogenic 
organisms on tracheal aspirate. The diagnosis of pneumonia was made if the patient met diagnostic criteria for tracheitis in addition to the development of a new pulmonary opacity on chest X-ray. Urinary tract infection was diagnosed if fever was present in the setting of increased white blood cells on urinalysis and a positive urine culture.

Baseline characteristics were assessed with descriptive statistics appropriate for the data. $\chi^{2}$ test was used to compare categorical data, and two-sample $t$-test or Mann-Whitney $U$-test were employed to compare continuous variables (normally or non-normally distributed, respectively). Those characteristics that were significantly different among groups were then chosen as candidate covariates.

Paired $t$-test was performed to compare CRP level changes before and after chest closure, whereas signed rank test was used to compare PCT levels right before and after chest closure as PCT was not normally distributed.

Pair-wise comparison over time or among surgical groups was performed to compare PCT or CRP levels using the ANOVA method. Bonferroni adjustment was applied for multiple comparisons in determining the statistical significance level.

For graphical presentation of the main outcomes, box-plots were generated for PCT and CRP from preoperation to up to $5 \mathrm{~d}$ post operation. PCT and CRP levels were also displayed with least square means plus $1 \mathrm{SD}$ over time by infection status.

Short-term surgical outcome data were inspected before multiple linear regression analysis. If the normal assumption did not hold for certain outcomes, then $\log 10$ based transformations were performed. No other data transformation was needed for this dataset. Spearman correlation was performed to screen candidate covariates other than surgical groups, PCT and CRP. Given the limited sample size, only highly significant variables were then selected into the model. SAS (SAS Institute, Cary, NC) PROC GLM (general linear model) was performed for multiple linear regression analysis, and $R^{2}$ was used to assess model fitting.

Longitudinal mixed model with repeated measurements was performed to model changes in PCT and CRP levels over time by infection status for those children requiring DSC. Various variance-covariance structures were tested for model fitting, and best models were chosen using Akaike information criteria with the smallest value. Time by infection status interaction was also tested in the model. Because both PCT and CRP were elevated at $24 \mathrm{~h}$ after surgery, in order to test whether the significance of time by infection interaction had happened postsurgery, the PCT and CRP at preoperation were omitted from the mixed model, and PCT or CRP levels were also compared at each postoperation time point. SAS PROC MIXED (mixed linear models) was utilized for this analysis. Surgical group was placed in all models to adjust for clinical differences.

\section{ACKNOWLEDGMENTS}

We thank Maureen Ishii and Edmund Orsini for their assistance in establishing laboratory protocols for the procalcitonin assay. We also recognize the nurses and staff of the cardiac intensive care unit and Clinical and Translational Research Centers at the Children's Hospital of Colorado for their valuable contributions to our project.

\section{STATEMENT OF FINANCIAL SUPPORT}

This work was supported by the National Institutes of Health $(\mathrm{NIH})$ Colorado Clinical and Translational Sciences Institute (grant UL1 RR025780). The contents are the authors' sole responsibility and do not necessarily represent official NIH views. Additional funding was obtained through the Brigid Hope Fund. Brahms-USA (Annapolis, MD) donated samples of the procalcitonin assay to the clinical laboratory at Children's Hospital Colorado for clinical or research use. These samples were then designated for use in this study by the clinical laboratory without additional direction from Brahms-USA.

Disclosure: All authors declare that they have no conflicts of interest.

\section{REFERENCES}

1. Kaplan JM, Wong HR. Biomarker discovery and development in pediatric critical care medicine. Pediatr Crit Care Med 2011;12:165-73.

2. Raja SG, Dreyfus GD. Modulation of systemic inflammatory response after cardiac surgery. Asian Cardiovasc Thorac Ann 2005;13:382-95.
3. Sponholz C, Sakr Y, Reinhart K, Brunkhorst F. Diagnostic value and prognostic implications of serum procalcitonin after cardiac surgery: a systematic review of the literature. Crit Care 2006;10:R145.

4. Arkader R, Troster EJ, Lopes MR, et al. Procalcitonin does discriminate between sepsis and systemic inflammatory response syndrome. Arch Dis Child 2006;91:117-20.

5. McMaster P, Park DY, Shann F, et al. Procalcitonin versus C-reactive protein and immature-to-total neutrophil ratio as markers of infection after cardiopulmonary bypass in children. Pediatr Crit Care Med 2009;10:21721.

6. Arkader R, Troster EJ, Abellan DM, et al. Procalcitonin and C-reactive protein kinetics in postoperative pediatric cardiac surgical patients. J Cardiothorac Vasc Anesth 2004;18:160-5.

7. Celebi S, Koner O, Menda F, et al. Procalcitonin kinetics in pediatric patients with systemic inflammatory response after open heart surgery. Intensive Care Med 2006;32:881-7.

8. Beghetti M, Rimensberger PC, Kalangos A, Habre W, Gervaix A. Kinetics of procalcitonin, interleukin 6 and C-reactive protein after cardiopulmonary-bypass in children. Cardiol Young 2003;13:161-7.

9. Dahaba AA, Metzler H. Procalcitonin's role in the sepsis cascade. Is procalcitonin a sepsis marker or mediator? Minerva Anestesiol 2009;75:447-52.

10. Becker KL, Snider R, Nylen ES. Procalcitonin in sepsis and systemic inflammation: a harmful biomarker and a therapeutic target. Br J Pharmacol 2010;159:253-64.

11. Skrak P, Kovacikova L, Kunovsky P. Procalcitonin, neopterin and C-reactive protein after pediatric cardiac surgery with cardiopulmonary bypass. Bratisl Lek Listy 2007;108:501-5.

12. Hammer S, Loeff M, Reichenspurner $\mathrm{H}$, et al. Effect of cardiopulmonary bypass on myocardial function, damage and inflammation after cardiac surgery in newborns and children. Thorac Cardiovasc Surg 2001;49:34954.

13. Séguéla PE, Joram N, Romefort B, et al. Procalcitonin as a marker of bacterial infection in children undergoing cardiac surgery with cardiopulmonary bypass. Cardiol Young 2011;21:392-9.

14. Nahum E, Schiller O, Livni G, Bitan S, Ashkenazi S, Dagan O. Procalcitonin level as an aid for the diagnosis of bacterial infections following pediatric cardiac surgery. J Crit Care 2012;27:220.e11-6.

15. Michalik DE, Duncan BW, Mee RB, et al. Quantitative analysis of procalcitonin after pediatric cardiothoracic surgery. Cardiol Young 2006;16:48-53.

16. Davidson J, Tong S, Hancock H, Hauck A, da Cruz E, Kaufman J. Prospective validation of the vasoactive-inotropic score and correlation to shortterm outcomes in neonates and infants after cardiothoracic surgery. Intensive Care Med 2012;38:1184-90.

17. Gaies MG, Gurney JG, Yen AH, et al. Vasoactive-inotropic score as a predictor of morbidity and mortality in infants after cardiopulmonary bypass. Pediatr Crit Care Med 2010;11:234-8.

18. Mofidi R, Suttie SA, Patil PV, Ogston S, Parks RW. The value of procalcitonin at predicting the severity of acute pancreatitis and development of infected pancreatic necrosis: systematic review. Surgery 2009;146:72-81.

19. Mann EA, Wood GL, Wade CE. Use of procalcitonin for the detection of sepsis in the critically ill burn patient: a systematic review of the literature. Burns 2011;37:549-58.

20. Tang H, Huang T, Jing J, Shen H, Cui W. Effect of procalcitonin-guided treatment in patients with infections: a systematic review and meta-analysis. Infection 2009;37:497-507.

21. Nahum E, Livni G, Schiller O, Bitan S, Ashkenazi S, Dagan O. Role of $\mathrm{C}$-reactive protein velocity in the diagnosis of early bacterial infections in children after cardiac surgery. J Intensive Care Med 2012;27:191-6.

22. Phua J, Koay ES, Lee KH. Lactate, procalcitonin, and amino-terminal pro-B-type natriuretic peptide versus cytokine measurements and clinical severity scores for prognostication in septic shock. Shock 2008;29:328-33.

23. Stammet P, Devaux Y, Azuaje F, et al. Assessment of procalcitonin to predict outcome in hypothermia-treated patients after cardiac arrest. Crit Care Res Pract 2011;2011:631062.

24. Los Arcos M, Rey C, Concha A, Medina A, Prieto B. Acute-phase reactants after paediatric cardiac arrest. Procalcitonin as marker of immediate outcome. BMC Pediatr 2008;8:18. 